\title{
The Difference in Sebum Secretion Affecting Development of Acne
}

\author{
In Soon Jung, Sook Jung Yun, Jee-Bum Lee \\ Department of Dermatology, Chonnam National University Medical School, Gwangju,Korea
}

Background: Although sebum secretion is crucial for acne development, acne lesion distribution is not always similar to the topographic differences of sebum secretion. Objective: To analyze whether sebum secretion affects acne development and distribution and to assess other factors possibly influencing the relationship between acne and sebum secretion. Methods: This single-center retrospective study included 67 acne patients and 50 controls. Acne patients were divided into 3 groups based on acne lesion distribution: T-zone dominant, U-zone dominant, and mixed groups. The secreted sebum level in each zone of acne patients was compared with that of controls. We also conducted correlation analysis between secreted sebum level and acne number, depending on the facial zone. Results: No significant difference was found between acne patients and controls regarding age and sex ratio. The $U$-zone dominant group showed increased sebum levels compared with controls in the U-zone and whole face, but a similar result was not obtained in the T-zone dominant group. Moreover, there was a significant correlation between the number of lesions and secreted sebum level in the U-zone, but not in the T-zone. Further, there was a more significant relation in the $U$-zone of male and young patients. Conclusion: We found that increased sebum secretion compared with the condition may

Received December 17, 2018, Revised March 5, 2019, Accepted for publication March 9, 2019

Corresponding author: Jee-Bum Lee, Department of Dermatology, Chonnam National University Medical School, 42 Jebong-ro, Dong-gu, Gwangju 61469, Korea. Tel: 82-62-220-6684, Fax: 82-62-222-4058, E-mail: jbmlee@ jnu.ac.kr

ORCID: https://orcid.org/0000-0002-1477-4037

This is an Open Access article distributed under the terms of the Creative Commons Attribution Non-Commercial License (http://creativecommons. org/licenses/by-nc/4.0) which permits unrestricted non-commercial use, distribution, and reproduction in any medium, provided the original work is properly cited.

Copyright (c) The Korean Dermatological Association and The Korean Society for Investigative Dermatology affect acne development, especially in the U-zone. Sex and age may also influence the relationship between acne and increased sebum secretion. Acne lesion distribution may vary from patient to patient because sebum secretion affects acne differently depending on multiple factors.

(Ann Dermatol 31(4) 426 433, 2019)

\section{-Keywords-}

Acne vulgaris, Sebum

\section{INTRODUCTION}

Acne is a very common condition of the pilosebaceous unit, usually affecting adolescents and young adults ${ }^{1}$. There have been many studies to find the factors affecting the acne development. The important pathophysiologic factors revealed so far are increased sebum secretion, altered follicular growth and differentiation, Propionibacterium acnes colonization in the follicle, and inflammation ${ }^{2-4}$. Sebum secretion has usually been considered as one of the main factors responsible for acne development ${ }^{5}$. It is generally accepted that patients with acne have increased sebum secretion compared with normal people $e^{6-8}$. Although sebum secretion is the key to the acne development, the distribution of acne lesions is not always similar to the topographic differences of sebum secretion ${ }^{9}$. The face can be divided into 2 zones. The T-zone includes the forehead, nose, and chin with higher sebum secretion, and the U-zone includes both cheeks with lower sebum secretion $^{10}$. However, not all acne patients have more acne lesions in the T-zone. Therefore, in this study, we checked whether facial sebum secretion really affects acne development and distribution and whether there are any other factors affecting the distribution. 


\section{MATERIALS AND METHODS}

\section{Subjects}

We retrospectively reviewed the secreted sebum level of patients with and without acne who visited our clinic from January 2014 to December 2017. Only subjects without dermatological diseases of the face, who checked the secreted sebum level of the face, were included in normal controls. In all, 67 patients with acne and 50 normal controls were enrolled. The 67 patients were divided into 3 groups depending on the distribution of acne lesions (Fig. 1). The patients whose acne lesions were located mainly in the T-zone were included in T-zone dominant group, and those whose acne lesions were located mainly in the $\mathrm{U}$-zone were included in $\mathrm{U}$-zone dominant group. The patients whose acne lesions were equally located in the $\mathrm{U}$-zone and T-zone were included in the mixed group ("equally located" meant that the difference between the number of acne in the U- and T-zones was less than $20 \%$ of the large value). Further, the patients aged less than 25 years were included in the young group, and those aged more than or equal to 25 years were included in the old group. Ethical approval was obtained from the ethnic committee of Chonnam National University Hospital (IRB no. CNUH-2018-134).

\section{Measurement of secreted sebum level}

All subjects washed their face and did not use any cosmetics or touch their face for 1 hour. After 1 hour, the sebum level on the skin surface was measured at 5 different sites on the face: the forehead, nose, chin, and both cheeks. The sebum level was checked using the Sebumeter ${ }^{\circledR}$ (SM815; C-K Electronics, Cologne, Germany). All measurements were taken by the same investigator and at constant conditions (temperature, $22^{\circ} \mathrm{C} \pm 2{ }^{\circ} \mathrm{C}$; humidity, $40 \% \pm 3 \%$ ). The sebum level in the T-zone, U-zone, and whole face was calculated as follows: mean sebum level on the forehead, nose, and chin; mean sebum level on both cheeks; and mean sebum level on the forehead, nose, chin, and both cheeks, respectively.

\section{Counting the number of acne lesions}

We reviewed the clinical photographs and counted the acne lesions. Clinical photographs were taken in 3 different views: anterior view, right lateral view, and left lateral view. Only inflammatory lesions such as papule, nodules, and cysts were counted. The acne lesions were independently evaluated by 2 researchers, and the average number of lesions counted by the 2 was calculated and used.

\section{Relation between sebum secretion and acne}

We tried to determine whether the secreted sebum level in the zones where acne lesions were mainly located was increased compared with that in the corresponding zones of normal controls; that is, the secreted sebum levels in the T-zone, U-zone, and whole face were compared with the secreted sebum levels in the same zones of normal controls. This analysis was conducted repeatedly for the T-zone dominant, U-zone dominant, and mixed groups. We also analyzed the correlation between the number of acne and the sebum level according to the facial zone in individual patients with acne.

\section{Statistical analysis}

Student's t-test and Mann-Whitney $U$ test were used for comparing the sebum level of acne patients and normal controls. Pearson's correlation test was used for correlation analysis between the secreted sebum level and the number of acne. All analyses were performed using PASW Statistics ver. 18.0 (IBM Corp., Armonk, NY, USA). A $p$-value of $<0.05$ was considered statistically significant.

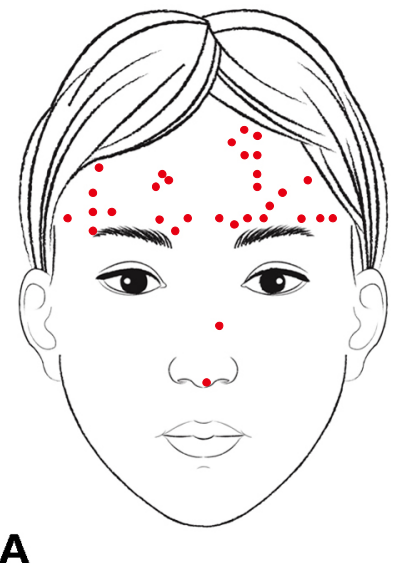

A

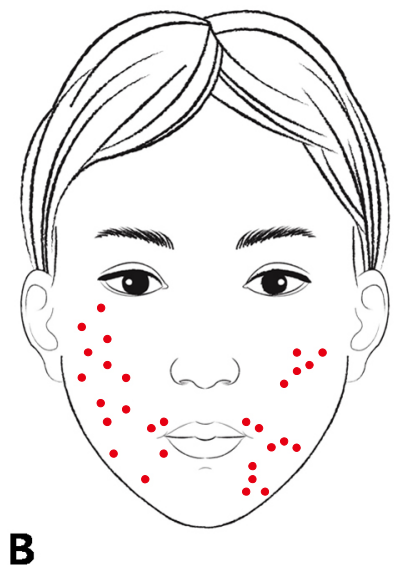

C

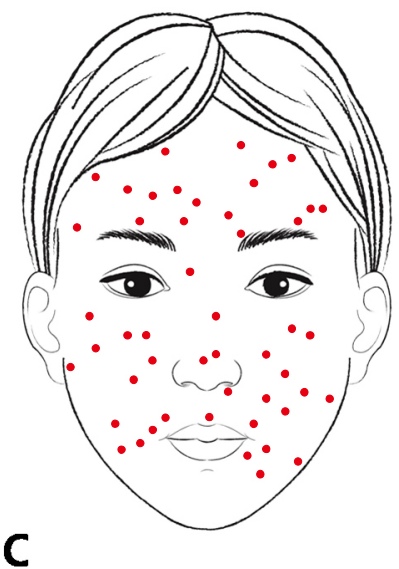

Fig. 1. Classification of patients with acne depending on the distribution of acne lesions. (A) T-zone dominant group, (B) U-zone dominant group, (C) mixed group. 


\section{RESULTS}

\section{Clinical data of patients and normal controls}

The clinical data of acne patients and normal controls for whom the sebum secretion was checked are summarized in Table 1. Of the 67 patients with acne, 39 were male, and 28 were female. Of the 50 normal controls, 25 were male, and 25 were female. The mean age of patients was $21.8 \pm 5.4$ years, and that of controls was $22.5 \pm 3.1$ years. Of the total, 53 patients and 31 normal controls were under 25 years of age, and 14 patients and 19 normal controls were over or equal to 25 years of age. There was no statistically significant difference between acne patients and normal controls with regard to mean age, sex ratio, and distribution according to age $(<25$ years or $\geq 25$ years). Three patients were included in the T-zone dominant group, 44 in the $U$-zone dominant group, and 20 in the mixed group. The mean age of patients in each group was $18.0 \pm 7.9$ years, $22.2 \pm 5.7$ years, and $21.6 \pm 4.2$ years, and the male-to-female ratio was 0:3, 1:0.57, and 1:0.82, respectively. There was no statistically significant difference between each patient group and control group in terms of mean age, sex ratio, and distribution according to age. The mean age was not significantly different between the male and female patients, and the male-to-female ratio was also not significantly different between the young and old patients.

\section{Sebum secretion affected acne development and distribution}

The comparison of the secreted sebum level between each patient group and the control group is summarized in Table 1. There was statistically significant difference in the secreted sebum level between the patients and the controls for all areas, the T-zone, U-zone, and whole face. In both patient group and control group, the secreted sebum level in the T-zone was higher than that in the $U$-zone. The sebum level in the T-zone was $77.7 \pm 60.1$, $100.5 \pm 71.3$, and $108.9 \pm 56.2$ in the T-zone dominant group, U-zone dominant group, and mixed group, respectively. A significant difference in the sebum secretion level in the T-zone was observed only between the mixed group and the control group $(80.6 \pm 39.1)(p<0.05)$. The sebum level in the $U$-zone was $24.7 \pm 26.1,78.1 \pm 78.3$, and $73.1 \pm 52.7$ in the T-zone dominant group, U-zone dominant group, and mixed group, respectively. In the $U$-zone, the $U$-zone dominant group and mixed group had more sebum secretion than the control group $(42.7 \pm 29.3)$ $(p<0.05)$. The sebum level in the whole face was $55.3 \pm$ $47.3,90.5 \pm 69.7$, and $94.1 \pm 47.7$ in the T-zone dominant group, U-zone dominant group, and mixed group, respectively. In the whole face, the $U$-zone dominant group and the mixed group show significantly higher levels of sebum secretion than the control group $(65.4 \pm 30.9)(p<0.05)$.

\section{Sex and age influenced the relation between acne development and sebum secretion}

The clinical data and secreted sebum level according to sex is summarized in Table 2. The sebum levels in the T-zone, U-zone, and whole face of male patients were significantly higher than those in the corresponding areas of female patients (T-zone: $p=0.036, U$-zone: $p=0.005$, whole face: $p=0.009$ ). Of the 39 male patients with acne, 28 patients were classified in the $U$-zone dominant group, 11 patients in the mixed group. Of the 28 female patients, 3 patients were classified in the T-zone dominant group, 16 in the U-zone dominant group, and 9 in the mixed group. In an analysis of 39 male patients and 25 male con-

Table 1. The clinical data of acne patients and normal controls for whom the sebum secretion was checked

\begin{tabular}{lccccc}
\hline \multicolumn{1}{c}{ Variable } & $\begin{array}{c}\text { T-zone dominant } \\
\text { group }\end{array}$ & $\begin{array}{c}\text { U-zone dominant } \\
\text { group }\end{array}$ & Mixed group & Total patients & Control group \\
\hline No. of patients & 3 & 44 & 20 & 67 & 50 \\
Male:female ratio & $0: 3$ & $1: 0.57$ & $1: 0.82$ & $1: 0.71$ & $1: 1$ \\
$\quad$ Male/female & $0 / 3$ & $28 / 16$ & $11 / 9$ & $39 / 28$ & $25 / 25$ \\
Age (yr) & $18.0 \pm 7.9$ & $22.2 \pm 5.7$ & $21.6 \pm 4.2$ & $21.8 \pm 5.4$ & $22.5 \pm 3.1$ \\
$<25$ & 2 & 34 & 17 & 53 & 31 \\
$\geq 25$ & 1 & 10 & 3 & 14 & 19 \\
Onset age (yr) & $14.0 \pm 7.4$ & $18.6 \pm 5.7$ & $19.3 \pm 5.5$ & $18.7 \pm 5.6$ & $\mathrm{~N} / \mathrm{A}$ \\
Secreted sebum level & & & & \\
T-zone & $77.7 \pm 60.1$ & $100.5 \pm 71.3$ & $108.9 \pm 56.2^{*}$ & $102.0 \pm 66.1^{*}$ & $80.6 \pm 39.1$ \\
U-zone & $24.7 \pm 26.1$ & $78.1 \pm 78.3^{*}$ & $73.1 \pm 52.7^{*}$ & $74.2 \pm 70.2^{*}$ & $42.7 \pm 29.3$ \\
Whole face & $55.3 \pm 47.3$ & $90.5 \pm 69.7^{*}$ & $94.1 \pm 47.7^{*}$ & $90.0 \pm 62.9^{*}$ & $65.4 \pm 30.9$ \\
\hline
\end{tabular}

Values are presented as number only or mean \pm standard deviation. N/A: not applicable. *Significantly different from control group $(p<0.05)$. 
Table 2. The clinical data of acne patients and normal controls according to sex (male or female)

\begin{tabular}{|c|c|c|c|c|c|}
\hline Variable & $\begin{array}{l}\text { T-zone dominant } \\
\text { group }\end{array}$ & $\begin{array}{l}\text { U-zone dominant } \\
\text { group }\end{array}$ & Mixed group & Total patients & Control group \\
\hline \multicolumn{6}{|l|}{ Male } \\
\hline No. of patients & 0 & 28 & 11 & 39 & 25 \\
\hline Age (yr) & $\mathrm{N} / \mathrm{A}$ & $21.5 \pm 5.7$ & $20.5 \pm 5.2$ & $21.3 \pm 5.5$ & $23.7 \pm 1.6$ \\
\hline$<25$ & $\mathrm{~N} / \mathrm{A}$ & 21 & 10 & 31 & 16 \\
\hline$\geq 25$ & $\mathrm{~N} / \mathrm{A}$ & 7 & 1 & 8 & 9 \\
\hline Onset age (yr) & N/A & $17.5 \pm 3.4$ & $18.0 \pm 7.7$ & $17.6 \pm 4.4$ & $\mathrm{~N} / \mathrm{A}$ \\
\hline \multicolumn{6}{|l|}{ Secreted sebum level } \\
\hline T-zone & $\mathrm{N} / \mathrm{A}$ & $112.1 \pm 81.2$ & $123.0 \pm 66.7$ & $115.1 \pm 76.7$ & $95.0 \pm 42.7$ \\
\hline U-zone & $\mathrm{N} / \mathrm{A}$ & $95.4 \pm 88.1^{*}$ & $84.7 \pm 65.1^{*}$ & $92.4 \pm 81.6^{*}$ & $45.6 \pm 33.5$ \\
\hline Whole face & $\mathrm{N} / \mathrm{A}$ & $104.8 \pm 80.0^{*}$ & $106.7 \pm 57.9^{*}$ & $105.3 \pm 65.3 *$ & $75.3 \pm 34.9$ \\
\hline \multicolumn{6}{|l|}{ Female } \\
\hline Number of patients & 3 & 16 & 9 & 28 & 25 \\
\hline Age (yr) & $18.0 \pm 7.9$ & $23.3 \pm 5.7$ & $22.8 \pm 2.2$ & $22.5 \pm 5.2$ & $21.4 \pm 3.8$ \\
\hline$<25$ & 2 & 13 & 7 & 22 & 16 \\
\hline$\geq 25$ & 1 & 3 & 2 & 6 & 9 \\
\hline Onset age (yr) & $14.0 \pm 9.9$ & $20.1 \pm 7.8$ & $20.6 \pm 1.9$ & $19.9 \pm 6.7$ & $\mathrm{~N} / \mathrm{A}$ \\
\hline \multicolumn{6}{|l|}{ Secreted sebum level } \\
\hline T-zone & $77.7 \pm 60.1$ & $80.2 \pm 44.8$ & $91.7 \pm 36.6^{*}$ & $83.6 \pm 42.6$ & $66.1 \pm 29.4$ \\
\hline U-zone & $24.7 \pm 26.1$ & $47.9 \pm 45.5$ & $59.0 \pm 29.5$ & $49.0 \pm 39.5$ & $39.8 \pm 24.6$ \\
\hline Whole face & $55.3 \pm 47.3$ & $65.5 \pm 37.1$ & $78.6 \pm 26.7^{*}$ & $68.6 \pm 34.6$ & $55.5 \pm 23.1$ \\
\hline
\end{tabular}

Values are presented as number only or mean \pm standard deviation. N/A: not applicable. *Significantly different from control group $(p<0.05)$.

Table 3. The clinical data of acne patients and normal controls according to age $(<25$ years or $\geq 25$ years $)$

\begin{tabular}{|c|c|c|c|c|c|}
\hline & $\begin{array}{l}\text { T-zone dominant } \\
\text { group }\end{array}$ & $\begin{array}{l}\text { U-zone dominant } \\
\text { group }\end{array}$ & Mixed group & Total patients & Control group \\
\hline \multicolumn{6}{|l|}{$<25$ years } \\
\hline No. of patients & 2 & 34 & 17 & 53 & 31 \\
\hline Male:female ratio & $0: 2$ & $1: 0.62$ & $1: 0.7$ & $1: 0.71$ & $1: 1.07$ \\
\hline Male/female & $0 / 2$ & $21 / 13$ & $10 / 7$ & $31 / 22$ & $15 / 16$ \\
\hline Age $(y r)$ & $13.5 \pm 2.1$ & $19.9 \pm 3.2$ & $20.4 \pm 2.8$ & $19.8 \pm 3.2$ & $20.8 \pm 2.6$ \\
\hline Onset age (yr) & $14.0 \pm 2.1$ & $17.0 \pm 3.4$ & $17.8 \pm 4.1$ & $17.1 \pm 3.5$ & $\mathrm{~N} / \mathrm{A}$ \\
\hline \multicolumn{6}{|c|}{ Secreted sebum level } \\
\hline T-zone & $43.4 \pm 12.8$ & $107.7 \pm 74.6$ & $113.1 \pm 60.2^{*}$ & $107.0 \pm 69.4$ & $78.9 \pm 38.2$ \\
\hline U-zone & $10.0 \pm 8.5$ & $83.3 \pm 83.5^{*}$ & $76.8 \pm 55.9^{*}$ & $78.4 \pm 74.8$ & $43.7 \pm 28.6$ \\
\hline Whole face & $28.2 \pm 8.5$ & $96.7 \pm 73.7^{*}$ & $98.0 \pm 50.7^{*}$ & $94.5 \pm 66.4$ & $64.8 \pm 28.9$ \\
\hline \multicolumn{6}{|l|}{$\geq 25$ years } \\
\hline No. of patients & 1 & 10 & 3 & 14 & 19 \\
\hline Male:female ratio & $0: 1$ & $1: 0.43$ & $1: 2$ & $1: 0.75$ & $1: 0.9$ \\
\hline Male/female & $0 / 1$ & $7 / 3$ & $1 / 2$ & $8 / 6$ & $10 / 9$ \\
\hline Age $(y r)$ & 27.0 & $29.9 \pm 5.5$ & $28.3 \pm 4.9$ & $29.4 \pm 5.1$ & $25.5 \pm 0.8$ \\
\hline Onset age (yr) & 25.0 & $24.4 \pm 8.8$ & $25.5 \pm 7.8$ & $24.7 \pm 8.1$ & $\mathrm{~N} / \mathrm{A}$ \\
\hline \multicolumn{6}{|c|}{ Secreted sebum level } \\
\hline T-zone & 146.3 & $76.0 \pm 54.7$ & $85.0 \pm 5.8$ & $83.0 \pm 49.3$ & $83.3 \pm 41.4$ \\
\hline U-zone & 54.0 & $60.7 \pm 57.0$ & $52.2 \pm 24.2$ & $58.4 \pm 48.5$ & $41.1 \pm 31.1$ \\
\hline Whole face & 109.4 & $69.4 \pm 51.8$ & $71.9 \pm 13.1$ & $72.8 \pm 44.7$ & $66.3 \pm 34.7$ \\
\hline
\end{tabular}

Values are presented as number only or mean \pm standard deviation. N/A: not applicable. ${ }^{*}$ Significantly different from control group $(p<0.05)$. 
trols, the U-zone dominant group had a significantly higher mean sebum secretion $(95.4 \pm 88.1)$ than the control group $(45.6 \pm 33.5)$ in the $U$-zone $(p<0.05)$, but not in T-zone $(112.1 \pm 81.2,95.0 \pm 42.7)$ and whole face $(104.8 \pm$ $80.0,75.3 \pm 34.9)$. The mixed group had a higher mean sebum secretion than the control group in the T-zone $(123.0 \pm 66.7,95.0 \pm 42.7)$, U-zone $(84.7 \pm 65.1,45.6 \pm$ $33.5)$, and whole face $(106.7 \pm 57.9,75.3 \pm 34.9)(p<0.05)$. In an analysis of 28 female patients and 25 female controls, mixed group showed higher sebum level than the control group in the T-zone $(91.7 \pm 36.6,66.1 \pm 29.4)$ and the whole face $(78.6 \pm 26.7,55.5 \pm 23.1)$. The T-zone dominant and $U$-zone dominant groups in female patients did not show statistically significant differences in any area compared with the corresponding areas in normal controls.

The clinical data and the secreted sebum level according to age are summarized in Table 3 . There was no statistically significant difference between young and old patients in all 3 zones. Of the 53 young patients with acne, 2 were classified in the T-zone dominant group, 34 in the $U$-zone dominant group, and 17 in the mixed group. Of the 14 old patients, 1 patient was classified in the T-zone dominant group, 10 in the U-zone dominant group, and 3 in the mixed group. In an analysis of 53 young patients and 31 young controls, the U-zone dominant group patients showed significantly higher sebum levels than control group individuals in the $\mathrm{U}$-zone and the whole face (U-zone: $83.3 \pm 83.5,43.7 \pm 28.6$, whole face: $96.7 \pm 73.7$, $64.8 \pm 28.9)(p<0.05)$, but not in the T-zone. The mixed

Table 4. The Pearson's correlation coefficients between the secreted sebum level and acne number in each zone according to sex and age difference

\begin{tabular}{|c|c|c|c|c|c|}
\hline & Total patients & Male & Female & $<25$ years & $\geq 25$ years \\
\hline $\begin{array}{l}\text { No. of the patients } \\
\mathrm{T} \text { zone }\end{array}$ & 67 & 39 & 28 & 53 & 14 \\
\hline $\begin{array}{l}\text { No. of lesion } \\
r \\
p \text {-value }\end{array}$ & $\begin{array}{c}13.2 \pm 9.6 \\
0.231(0.060)\end{array}$ & $\begin{array}{c}11.2 \pm 9.5 \\
0.310(0.055)\end{array}$ & $\begin{array}{c}16.1 \pm 9.1 \\
0.383(0.044) *\end{array}$ & $\begin{array}{c}13.9 \pm 9.8 \\
0.292(0.034) *\end{array}$ & $\begin{array}{c}10.6 \pm 8.7 \\
-0.148(0.614)\end{array}$ \\
\hline $\begin{array}{l}\text { Forehead } \\
\text { No. of lesion } \\
r \\
p \text {-value }\end{array}$ & $\begin{array}{c}6.7 \pm 7.0 \\
0.150(0.224)\end{array}$ & $\begin{array}{c}5.7 \pm 6.8 \\
0.129(0.434)\end{array}$ & $\begin{array}{c}8.1 \pm 7.2 \\
0.212(0.278)\end{array}$ & $\begin{array}{c}7.1 \pm 7.5 \\
0.194(0.163)\end{array}$ & $\begin{array}{c}5.3 \pm 4.7 \\
-0.162(0.579)\end{array}$ \\
\hline $\begin{array}{l}\text { Nose } \\
\text { No. of lesion } \\
r \\
p \text {-value }\end{array}$ & $\begin{array}{c}1.5 \pm 1.6 \\
0.198(0.110)\end{array}$ & $\begin{array}{c}1.6 \pm 1.8 \\
0.264(0.110)\end{array}$ & $\begin{array}{c}1.5 \pm 1.4 \\
0.007(0.973)\end{array}$ & $\begin{array}{c}1.7 \pm 1.7 \\
0.200(0.156)\end{array}$ & $\begin{array}{c}1.1 \pm 1.3 \\
-0.043(0.885)\end{array}$ \\
\hline $\begin{array}{l}\text { Chin } \\
\text { No. of lesion } \\
r \\
p \text {-value }\end{array}$ & $\begin{array}{c}5.0 \pm 4.0 \\
0.059(0.682)\end{array}$ & $\begin{array}{c}3.9 \pm 3.5 \\
0.038(0.841)\end{array}$ & $\begin{array}{c}6.5 \pm 4.2 \\
0.502(0.024) *\end{array}$ & $\begin{array}{c}5.1 \pm 4.0 \\
0.074(0.649)\end{array}$ & $\begin{array}{c}4.3 \pm 4.1 \\
-0.162(0.656)\end{array}$ \\
\hline $\begin{array}{l}\text { U zone } \\
\text { No. of lesion } \\
\mathrm{R} \\
p \text {-value }\end{array}$ & $\begin{array}{c}24.1 \pm 14.7 \\
0.362(0.003)^{*}\end{array}$ & $\begin{array}{c}25.3 \pm 16.5 \\
0.335(0.037)^{*}\end{array}$ & $\begin{array}{c}22.4 \pm 11.8 \\
0.432(0.022)^{*}\end{array}$ & $\begin{array}{c}25.0 \pm 13.9 \\
0.482(0.000)^{*}\end{array}$ & $\begin{array}{c}20.9 \pm 17.6 \\
-0.221(0.448)\end{array}$ \\
\hline $\begin{array}{l}\text { Right cheek } \\
\text { No. of lesion } \\
r \\
p \text {-value }\end{array}$ & $\begin{array}{c}12.1 \pm 7.8 \\
0.349(0.004)^{*}\end{array}$ & $\begin{array}{c}12.9 \pm 8.6 \\
0.356(0.026)^{*}\end{array}$ & $\begin{array}{c}11.1 \pm 6.5 \\
0.389(0.041)\end{array}$ & $\begin{array}{c}12.8 \pm 7.5 \\
0.431(0.001)^{*}\end{array}$ & $\begin{array}{c}9.6 \pm 8.5 \\
-0.117(0.691)\end{array}$ \\
\hline $\begin{array}{l}\text { Left cheek } \\
\text { No. of lesion } \\
r \\
p \text {-value }\end{array}$ & $\begin{array}{c}12.0 \pm 7.5 \\
0.304(0.012)^{*}\end{array}$ & $\begin{array}{c}12.4 \pm 8.3 \\
0.248(0.128)\end{array}$ & $\begin{array}{c}11.4 \pm 6.1 \\
0.305(0.115)\end{array}$ & $\begin{array}{c}12.2 \pm 6.9 \\
0.434(0.001)^{*}\end{array}$ & $\begin{array}{c}11.3 \pm 9.6 \\
-0.118(0.687)\end{array}$ \\
\hline $\begin{array}{l}\text { Whole face } \\
\text { No. of lesion } \\
r \\
p \text {-value }\end{array}$ & $\begin{array}{c}37.3 \pm 20.6 \\
0.326(0.007)^{*}\end{array}$ & $\begin{array}{c}36.5 \pm 22.2 \\
0.355(0.026)^{*}\end{array}$ & $\begin{array}{c}38.6 \pm 18.4 \\
0.470(0.012)^{*}\end{array}$ & $\begin{array}{c}38.9 \pm 19.6 \\
0.468(0.000) *\end{array}$ & $\begin{array}{c}31.5 \pm 23.8 \\
-0.322(0.262)\end{array}$ \\
\hline
\end{tabular}

r: correlation coefficient. *Significant correlation at the $5 \%$ level. 
group showed higher sebum level than the control group in the T-zone $(113.1 \pm 60.2,78.9 \pm 38.2)$, U-zone $(76.8 \pm$ $55.9,43.7 \pm 28.6)$, and whole face $(98.0 \pm 50.7,64.8 \pm 28.9)$ $(p<0.05)$. In an analysis of 14 old patients and 19 old controls, no group showed a statistically significant difference in any zone compared with normal controls

\section{Increased sebum secretion had a positive correlation with the number of acne lesions}

The results of correlation analyses between the secreted sebum level and the number of acne lesions are summarized in Table 4. The acne lesions were located more in the $U$-zone $(24.1 \pm 14.7)$ than in the T-zone $(13.2 \pm 9.6)$. The secreted sebum level showed a positive correlation with the number of acne lesions in the $U$-zone $(r=0.362$, $p=0.003)$ and the whole face $(r=0.326, p=0.007)$.

In both male and female patients, acne lesions were located more in the $\mathrm{U}$-zone than in the T-zone. In the 39 male patients, secreted sebum level showed statistically significant correlation with the number of acne lesions in the $U$-zone $(r=0.335, p=0.037)$ and the whole face $(r=0.355, p=0.026)$. In the 28 female patients, the sebum secretion level in the T-zone $(r=0.383, p=0.044), U$-zone $(r=0.432, \quad p=0.022)$, and the whole face $(r=0.470$, $p=0.012$ ) showed a positive correlation with the number of acne lesions.

In both young and old patients, acne lesions were located more in the $U$-zone than in the T-zone. In the 53 young patients, the T-zone $(r=0.292, p=0.034)$, $U$-zone $(r=0.482$, $p=0.000)$, and the whole face $(r=0.468, p=0.000)$ had a statistically positive correlation with the number of acne lesions. However, in the 14 old patients, there was no significant correlation in all 3 areas.

\section{DISCUSSION}

The sebum secretion on the face varies depending on the topographical differences ${ }^{11}$. The distribution of acne lesions and the topographical difference in sebum secretion are not always similar. This raises the question of whether sebum secretion actually affects acne development and distribution.

In our analysis, we demonstrated that increased sebum secretion affected acne development. The results of student's t-test showed that the secreted sebum level of patients with acne was increased compared with that of the control group, and the results of correlation analysis showed that the number of acne lesions tended to increase as the sebum level increased. We also found that sebum secretion affected acne differently depending on the location of the acne lesions. The U-zone dominant group showed a higher sebum level than the normal controls in the $U$-zone, but not in the T-zone. However, T-zone dominant group did not show increased sebum secretion compared with normal controls in the T-zone or the U-zone. Additionally, there was a significant correlation between the number of lesions and the secreted sebum level in the U-zone, but not in the T-zone. With these results, we suggest that increased sebum secretion especially affected acne development in the U-zone. One of the previous studies that analyzed the correlation between lesion distribution and topographical differences in facial sebum reported that increased sebum secretion did not have a direct relationship with the number of acne lesions $^{12}$. The authors suggested that increased facial sebum was not the primary cause of acne, but just an epiphenomenon. However, another study showed statistically significant correlations between the casual sebum level and the number of inflammatory and acne lesions ${ }^{13}$. These authors performed the correlation analysis in the same manner as we did in our study. The correlation was more significant in the $U$-zone than in the T-zone, which was similar to our results. The reason why the results differ according to the analysis is not exactly clear. Recently, it has been reported that the content ratio of sebum, rather than the total amount of sebum, affects acne development ${ }^{7,14}$. In our study, the content of sebum was not analyzed; therefore, the effect of sebum content on acne development was not considered.

Sex and age also influenced the relation between acne development and sebum secretion. In male patients, increased sebum secretion only in the $U$-zone affected acne development in both results. The $U$-zone dominant group showed higher sebum level than normal controls in the $U$-zone and whole face, and mixed group showed higher sebum level than normal controls in the U-zone and whole face. There was also a significant correlation between the number of lesions and secreted sebum level in the $U$-zone and whole face. As a result, in male patients, increased sebum secretion in the U-zone may have a greater effect on acne development than that in the T-zone. In female patients, the U-zone dominant and T-zone dominant groups did not show increased sebum secretion levels than the control group in the $U$-zone and T-zone, respectively. However, there was a significant correlation between the number of lesions and secreted sebum level in the T-zone, $\mathrm{U}$-zone, and whole face. These 2 results are conflicting, and therefore, we did not find any zone that affected the relation between acne and sebum secretion in female patients. With respect to sex, our results suggested that sex difference influenced the relationship between acne development and increased sebum secretion. 
With regard to young patients, the $U$-zone dominant group showed increased sebum secretion compared with the normal controls in the U-zone and whole face, and the mixed group showed increased sebum secretion compared with the normal controls in the T-zone, $\mathrm{U}$-zone, and whole face. Additionally, there was a significant correlation between the number of lesions and secreted sebum level in the T-zone, $U$-zone, and whole face. Increased sebum secretion in the U-zone may affect acne development in both results. In old patients, we did not find any significant results. These findings suggested that age also influenced the relationship between the acne development and increased sebum secretion. Because sebum secretion affects acne differently depending on various factors such as location of acne lesions, sex, and age, the distribution of acne lesions may vary from patient to patient. This finding is supported by the fact that acne presents different clinical features in different age groups and sexes. For example, adult female acne, defined as acne in female over 25 years of age, is considered as a specific subtype ${ }^{15}$. The reason why an increased sebum level affects acne differently depending on age and sex is unclear. Many factors such as hormonal fluctuations, genetics, cosmetics, diet, and stress may be attributed ${ }^{16,17}$.

There were some limitations to this study. First, Sample size of the T-zone dominant group was small. Further research is required with a large number of patients. When we counted the number of acne lesions, we checked only inflammatory lesions. The sebum level was checked 1 hour after cleansing considering many factors. Therefore, the amount of sebum secretion in patients and normal control was relatively lower than that reported in previous studies. However, all patients and normal controls were checked under the same conditions, which would result in no effect on the analysis of correlation. Secreted sebum level was checked at just 5 different points; therefore, the measured amount of sebum may not accurately reflect the amount of sebum in the entire area. The amount of secreted sebum can be measured differently according to seasonal change. Youn et al. $^{10}$ reported that the face showed seasonal variations in sebum secretion. The facial sebum excretion for summer was statistically higher than that for other seasons. In this study, secreted sebum level was not checked at the same season. Sixteen out of 67 patients with acne $(23.9 \%)$ were checked in summer, while 10 out of 50 normal controls $(20.0 \%)$ were checked in summer.

In conclusion, the increased sebum level compared with the normal condition may affect acne development, especially in the U-zone. Sex and age may also influence the correlation of acne with increased sebum secretion.
Because sebum secretion affects acne differently depending on multiple factors such as location of acne lesions, sex, and age, the distribution of acne lesions may vary from patient to patient. Further studies are required to clarify the pathogenesis of acne with different distributions. Such studies can help improve the diagnosis and treatment according to the appropriate cause and condition.

\section{CONFLICTS OF INTEREST}

The authors have nothing to disclose.

\section{ORCID}

In Soon Jung, https://orcid.org/0000-0002-0548-7159

Sook Jung Yun, https://orcid.org/0000-0003-4229-5831

Jee-Bum Lee, https://orcid.org/0000-0002-1477-4037

\section{REFERENCES}

1. Leyden JJ. A review of the use of combination therapies for the treatment of acne vulgaris. J Am Acad Dermatol 2003;49(3 Suppl):S200-S210.

2. Strauss JS, Krowchuk DP, Leyden JJ, Lucky AW, Shalita AR, Siegfried EC, et al. Guidelines of care for acne vulgaris management. J Am Acad Dermatol 2007;56:651-663.

3. Gollnick H, Cunliffe W, Berson D, Dreno B, Finlay A, Leyden JJ, et al. Management of acne: a report from a Global alliance to improve outcomes in acne. J Am Acad Dermatol 2003;49(1 Suppl):S1-S37.

4. Zouboulis CC, Eady A, Philpott M, Goldsmith LA, Orfanos C, Cunliffe WC, et al. What is the pathogenesis of acne? Exp Dermatol 2005;14:143-152.

5. Li X, He C, Chen Z, Zhou C, Gan Y, Jia Y. A review of the role of sebum in the mechanism of acne pathogenesis. J Cosmet Dermatol 2017;16:168-173.

6. Zouboulis CC. Acne and sebaceous gland function. Clin Dermatol 2004;22:360-366.

7. Pappas A, Johnsen S, Liu JC, Eisinger M. Sebum analysis of individuals with and without acne. Dermatoendocrinol 2009;1:157-161.

8. Burton JL, Shuster S. The relationship between seborrhoea and acne vulgaris. Br J Dermatol 1971;85:197-198.

9. Youn SW. The role of facial sebum secretion in acne pathogenesis: facts and controversies. Clin Dermatol 2010;28:8-11.

10. Youn SW, Na JI, Choi SY, Huh CH, Park KC. Regional and seasonal variations in facial sebum secretions: a proposal for the definition of combination skin type. Skin Res Technol 2005;11:189-195.

11. Youn SH, Choi CW, Choi JW, Kim BR, Byun SY, Youn SW. Novel facial cosmetic area 'O zone' shows unique characteristics in sebum excretion and acne lesion distribution. Skin Res Technol 2014;20:164-169.

12. Youn SW, Park ES, Lee DH, Huh CH, Park KC. Does facial 
sebum excretion really affect the development of acne? $\mathrm{Br}$ J Dermatol 2005;153:919-924.

13. Choi CW, Choi JW, Park KC, Youn SW. Facial sebum affects the development of acne, especially the distribution of inflammatory acne. J Eur Acad Dermatol Venereol 2013;27:301-306.

14. Ottaviani M, Camera E, Picardo M. Lipid mediators in acne. Mediators Inflamm 2010;2010:858176.
15. Zeichner JA, Baldwin HE, Cook-Bolden FE, Eichenfield LF, Fallon-Friedlander S, Rodriguez DA. Emerging issues in adult female acne. J Clin Aesthet Dermatol 2017;10:37-46.

16. Preneau S, Dreno B. Female acne-a different subtype of teenager acne? J Eur Acad Dermatol Venereol 2012;26: 277-282.

17. Shaw JC, White LE. Persistent acne in adult women. Arch Dermatol 2001;137:1252-1253. 